\title{
Entrapment of Streptomycete Spores in a Chitosan-Polyphosphate Matrix
}

\author{
Guy Jobin, Gilles Grondin and Carole Beaulieu
}

Université de Sherbrooke, Faculté des Sciences, Département de biologie, Groupe de recherche sur l'étude et la valorisation de la diversité microbienne, Sherbrooke (Québec) Canada J1K 2R1

Drawing on the knowledge gained in the encapsulation technique called complex coacervation, an experiment was conducted in order to assess the faisability of successfully entrapping spores of a streptomycete strain in beads of chitosan-polyphosphate. Chitosan is a biopolymer endowed with worthwhile physico-chemical characteristics for use in forming hydrogels with polyanionic counterions (Kas, 1997). This matrix was already used for the preparation of gel beads and controlled release of an anticancer drug (Mi et al., 1999a, b) and for the entrapment of microbial cells (Vorlop and Klein, 1981, 1987). The chitosan-polyphosphate complex has also been used as a slow-release phosphate fertilizer (Frossard et al., 1994).

Microscopic techniques were used to characterize the beads. Thin sections of these beads were colored with toluidine blue in order to reveal the distribution of spores as well as the microfibril network of the chitosan-polyphosphate matrix. Thereafter, the dye live/dead evidenced the viability of the entrapped spores. Phase-contrast and interferential-contrast microscopy served to characterize further the network of microfibrils.

On figure 1 appears a $3-\mu \mathrm{m}$ section of the entrapped spores in a bead of chitosan $1 \%$ stained with toluidine blue.

References

Frossard, E. Tekely, P. and J.L. Morel. 1994. Chemical characterization and agronomic effectiveness of phosphorus applied as a polyphosphate-chitosan complex. Fertilizer Research. 37 : 151-158.

Kas, H.S. 1997. Chitosan : properties, preparation and application to microparticulate systems. J. Microencapsulation. 14 (6) : 689-711.

Mi, F.-L. et al., 1999a. Chitosan-Polyelectrolyte complexation for the preparation of gel beads and controlled release of anti-cancer drug. I. Effect of phosphorus Polyelectrolyte Complex and Enzymatic Hydrolysis of Polymer. Journal of Applied Polymer Science. 74 : 1868-1879.

Mi, F.-L. et al., 1999b. Chitosan-Polyelectrolyte complexation for the preparation of gel beads and controlled release of anti-cancer drug. II. Effect of pH-dependent ionic crosslinking or interpolymer complex using tripolyphosphate or polyphosphate as reagent. Journal of Applied Polymer Science. 74 : 1093-1107.

Vorlop, K.-D. and J. Klein. 1981. Formulation of spherical chitosan biocatalysts by ionotrophic gelation. Biotechnology Letters. 3 (1) : 9-14.

Vorlop, K.-D. and J. Klein. 1987. Entrapment of microbial cells in chitosan. In : Methods in Enzymology. 135 : 259-268. 


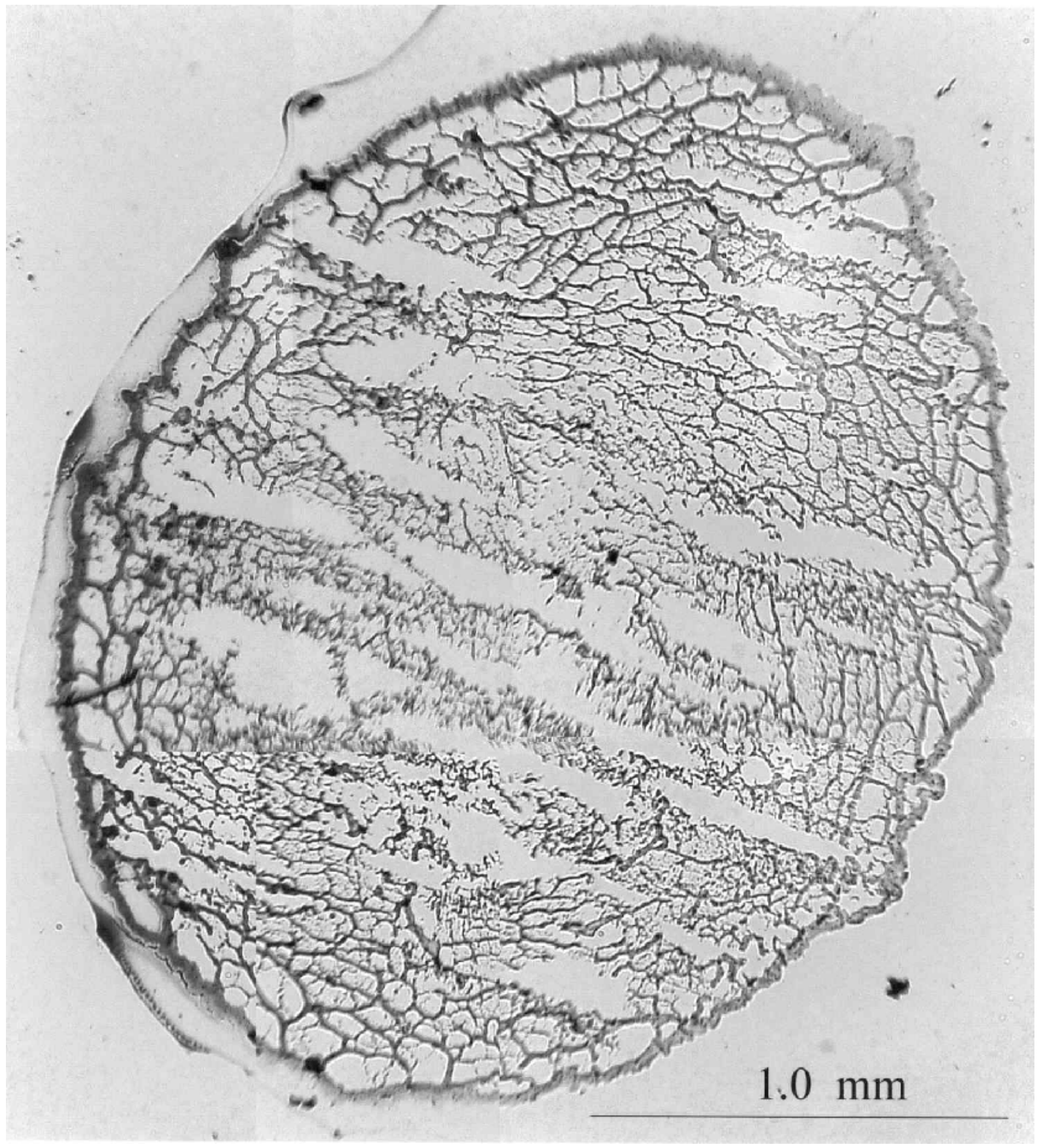

Figure 1: Section of $3 \mu \mathrm{m}$. Streptomycete Spores entrapped in a bead of chitosan $1 \%$ stained with toluidine blue 